13 years post OLT. However, from this point onwards mean plasma SA remained elevated with a low-normal PBG synthase activity similar to that seen in nitisinone treated patients. There was no apparent correlation between SA levels and renal function. Despite reduced $\mathrm{PBG}$ synthase levels, no patients developed porphyria-like symptoms.

Conclusion Urinary and plasma SA levels are elevated following OLT for TT1. Low-normal PBG synthase activity suggests the circulating SA may be functional. The clinical significance of this is unclear.

\section{P80 PATIENT CHARACTERISTICS AND OUTCOMES IN A 'HUB AND SPOKE MODEL' FOR LIVER TRANSPLANTATION PROVISION: THE SOUTH WEST LIVER UNIT/KING'S COLLEGE EXPERIENCE}

doi:10.1136/gutjnl-2011-300857a.80

${ }^{1}$ M Petrova, M Fung, M E Cramp, J D Mitchell, T J S Cross. ${ }^{1}$ The South West Liver Unit, Derriford Hospital, Plymouth, Devon, UK

Introduction Liver transplantation (LTx) is the only curative therapeutic modality for patients with end-stage liver disease (ESLD). A detailed evaluation of the liver transplant patient is critical to identify patients most likely to benefit from LTx in an era of organ donor shortage to optimise use of a scarce resource.

Aim The aim of this study was to analyse the profile and outcome of LTx referrals in a 'hub and spoke' LTx service.

Method A retrospective study of all patients referred to the South West Liver Unit, liver transplant service between April 2007 and April 2011. Patients with acute liver failure were excluded from the analysis. Pre-transplant and post-operative follow-up was performed at the South West Liver Unit. All operations were performed at King's College Hospital, London. Pre-LTx demographic and laboratory data were analysed using descriptive methods. Comparisons (Mann-Whitney) and survival (Kaplan-Meier) were estimated. $\alpha$ level of 0.05 was accepted as significant.

Results 191 consecutive patients ( $n=128,67 \%$ males) underwent elective pretransplant assessment and posttransplant management. Mean age was 53 years, SD 10.3 (range 19-70). Currently, 9.4\% (18/ 191) patients are under assessment, $10.5 \%(20 / 191)$ are on the waiting list for LTx, 29.8\% (57/191) have been transplanted, $7.9 \%$ $(15 / 191)$ died on the list and $42.4 \%(81 / 191)$ were assessed but not listed (too advanced disease in $12 \%$, not fulfilling minimal listing criteria $14.7 \%$ and contraindicated $15.7 \%$ ). Among patients who met minimal listing indications $21 \%(n=19)$ were diagnosed with

\section{Survival of all assessed patients}

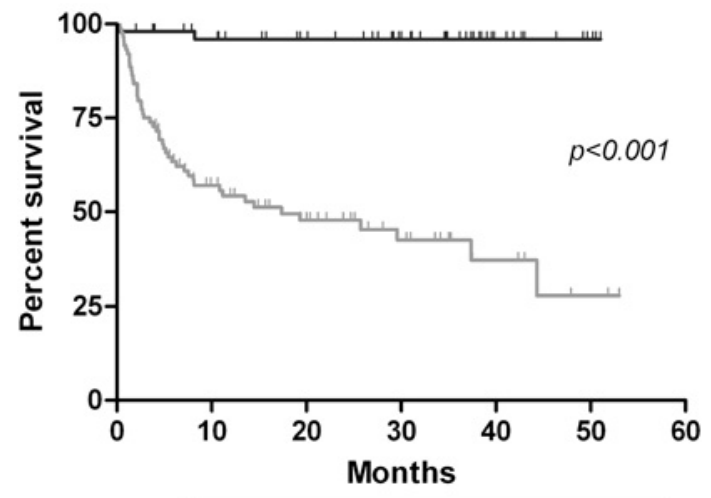

$\leftarrow$ LTX patients $\longrightarrow$ Not transplanted

Abstract P80 Figure 1 Survival of all patients. hepatocellular carcinoma (chronic HCV infection or/and alcohol background), 30\% ( $n=28)$ had alcoholic liver disease, 9\% $(n=8)-$ chronic HCV infection, $11 \%(\mathrm{n}=10)$ exhibit both alcohol and viral aetiology, 4\% ( $n=4)$-autoimmune hepatitis, 11\% ( $n=10)$ - PBC/ PSC, $4 \%$ NASH $(n=4), 2 \%(n=2)$ cryptogenic cirrhosis and in $9 \%$ $(n=8)$ rare diseases (vascular, metabolic, congenital or chronic rejection). These proportions did not deviate from the whole assessed cohort. Mean UKELD, MELD and CTP scores of all assessed patients were 52 (SD 5.2), 12 (SD 5.6) and 8 (SD 1.9). UKELD correlated strongly with MELD and CTP (Spearman's $\rho 0.68$ and $0.72, \mathrm{p}<0.01)$ and was slightly higher in listed for LTx group. CTP score did not differ between transplanted and not transplanted patients. Among the liver recipients $36 \%$ were blood group A, $12 \%$ $\mathrm{B}, 10 \% \mathrm{AB}$ and $42 \% \mathrm{O}$, similar to the distribution in the whole group. Mean BMI was 26.2, not different between transplanted and not listed patients. However, a third of all assessed patients had severe protein malnutrition, evaluated with hand dynamometry and estimated energy expenditure/intake ratio. The prevalence of HPS and $\mathrm{PPH}$ were $9 \%(11 / 122)$ and $3 \%(4 / 135)$ respectively. Three months-, 1- and 3-year survival of the patients and the grafts were $98 \% / 97 \% / 97 \%$ and $98 \% / 95 \% / 90 \%$. Abstract P80 figure 1 illustrates the differences in survival of transplanted and not transplanted patients.

Conclusion Graft and patient survival in the 'hub and spoke' model is good. Alcohol and hepatoma are the commonest reasons for listing. Protein malnutrition is common in this patient cohort suggesting improved patient nutrition and early dietician involvement is needed.

\section{P81 PRE-TRANSPLANT HISTOLOGICAL ASSESSMENT IN PATIENTS WITH ALCOHOLIC LIVER DISEASE DOES NOT PREDICT RISK OF RECIDIVISM POST LIVER TRANSPLANTATION}

doi:10.1136/gutjnl-2011-300857a.81

${ }^{1} \mathrm{~J}$ Prentis, ${ }^{2} \mathrm{~S}$ McPherson, ${ }^{3} \mathrm{~A}$ Burt, ${ }^{2} \mathrm{D}$ Manas, ${ }^{1} \mathrm{C}$ Snowden, ${ }^{2} \mathrm{M}$ Hudson, ${ }^{2} \mathrm{~S}$ Masson. ${ }^{1}$ Department of perioperative and critical care medicine, Freeman Hospital; ${ }^{2}$ Liver transplant Unit, Freeman Hospital; ${ }^{3}$ Institute of cellular medicine, Newcastle University

Introduction Liver transplantation for alcoholic liver disease (ALD) is well established, but it remains a controversial indication. Assessment of patients in whom alcohol has been a contributing factor to chronic liver disease aims to identify those at risk of relapse. As well as psychological assessment, histological evidence of steatohepatitis may indicate ongoing alcohol consumption.

Aim To determine the prevalence of histological evidence of ongoing alcohol use among patients undergoing liver transplant assessment for ALD and its affect on listing and risk of recidivism.

Method Consecutive patients with ALD assessed for liver transplantation between 2006 and 2010 were included. Transjugular liver biopsy was performed routinely in all patients and specimens were reviewed by an expert hepatopathologist. Outcomes including decision to list for transplant, survival and recidivism (patient admission or blood alcohol detection) were recorded

Results 122 patients were included (70\% male, median age 55) with diagnoses of lone ALD (92), ALD/HCV (10) and HCC (10) Histology was insufficient for analysis in four. Most patients (108, $89 \%$ ) had histological evidence of micronodular cirrhosis without evidence of steatosis or steatohepatitis, suggesting abstinence. Of these, 52 were listed for transplantation though two were subsequently removed for recidivism. To date, 39 have been transplanted; 1 -year follow-up was available in 28. The overall 1-year survival was $90 \%$ and $28 \%$ had returned to drinking alcohol $(n=7)$, although none were 'problem' drinkers. 